\title{
Bivalirudin surpasses heparin as treatment for myocardial infarction
}

A major new study led by Gregg W Stone, professor of medicine at Columbia University Medical Center, NY, USA, has demonstrated that direct treatment with the thrombin inhibitor bivalirudin offers a significantly improved clinical outcome for heart attack patients compared with those treated with heparin. Patients with ST-segment elevation myocardial infarction (STEMI), undergoing treatment with bivalirudin during primary angioplasty, suffered significantly fewer adverse ischemic events as a result of less severe bleeding, and fewer major cardiovascular events.

The study was a prospective randomized trial conducted across 11 countries, involving 3602 patients admitted within $12 \mathrm{~h}$ of a STEMI event. Patients were randomly assigned to either treatment with heparin plus a glycoprotein IIb/IIIa

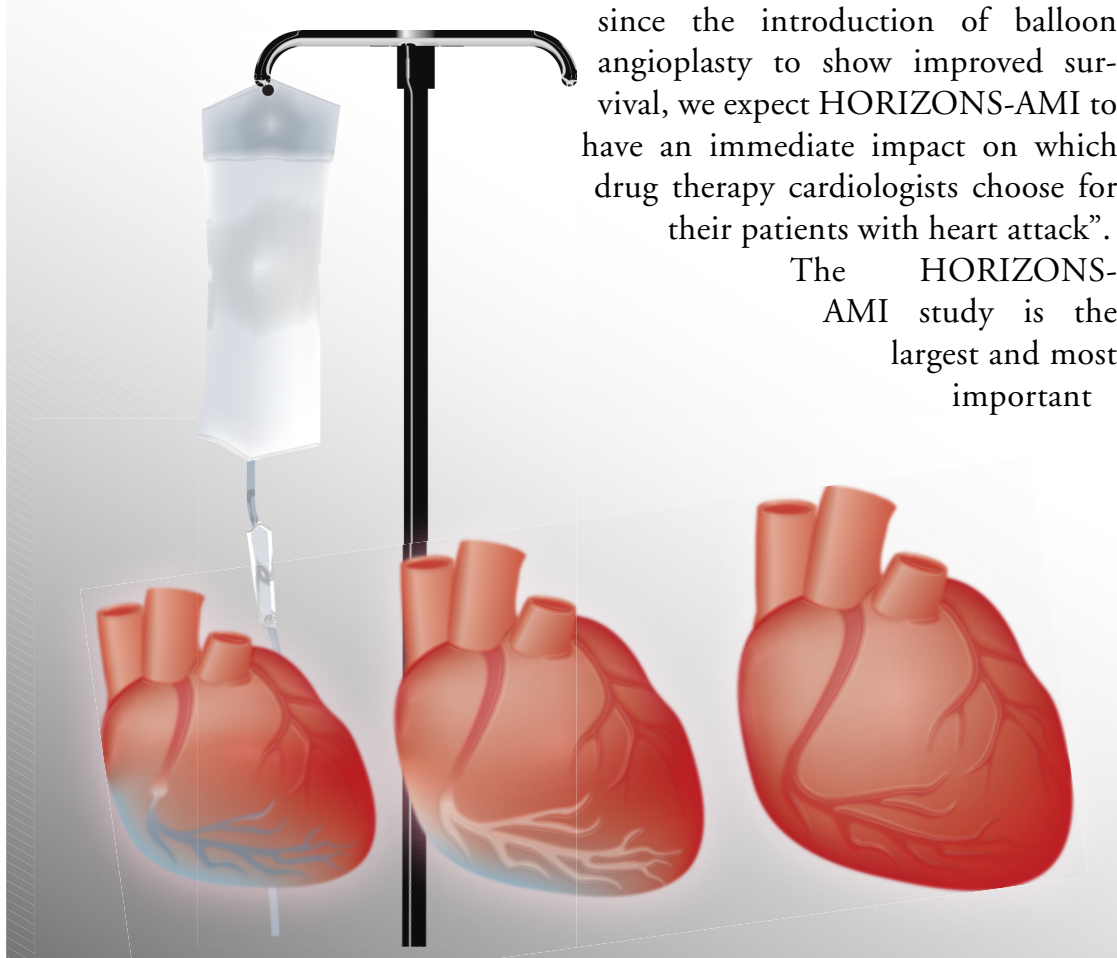

primary research to date on the use of

(GPI) inhibitor or treatment using bivalirudin alone; with clinical outcomes reported during and at 30 days. The results of the trial show that in those patients treated with bivalirudin, a $37.9 \%$ reduction in death from cardiac causes (cardiac mortality) was observed, a $40 \%$ reduction in major bleeding (a chief precursor of death in angioplasty patients), and a $24 \%$ net reduction in adverse clinical events occurred. In addition to this, when compared to treatment with heparin and GPI, overall mortality rates fell by $33 \%$.

Stone said of these significant findings, "The HORIZONS-AMI data show that using bivalirudin instead of heparin with a GPI during angioplasty increases survival in heart attack patients who are at high risk for death or disability ... As the first multicenter randomized primary angioplasty trial since the introduction of balloon angioplasty to show improved survival, we expect HORIZONS-AMI to have an immediate impact on which treatment. The use of bivalirudin for
patients of primary PCI is thus developamongst clinicians, not least for its
major impact on the event-free survival amongst clinicians, not least for its
major impact on the event-free survival of this foremost affliction.

\footnotetext{
Source: Stone GW, Witzenbichler B, Guagliumi G et al:; HORIZONS-AMI Trial Investigators: Bivalirudin during primary PCI in acute myocardial infarction. N. Engl. J.
Med. 358(21), 2218-2230 myocardial infarction. N. Engl. J.
Med. 358(21), 2218-2230
} (2008). treatment. The use of bivalirudin for
patients of primary PCI is thus developanticoagulant therapy for cardiac patients with STEMI who are undergoing angioplasty. One of the most significant findings relates to the huge reduction in bleeding following surgery that leads to major hemorfhagic complications, which is a key
factor in the number of overall deaths from myocardial infarction each year. Stone says of this discovery, "In multiple previous trials, major bleeding has been shown to be a strong predictor of short- and long-term mortality in patients undergoing angioplasty and in those with acute coronary syndromes", highlighting the significance of the finding.

It has also been reported that bivalirudin has already commenced use as a treatment option following primary percutaneous coronary intervention (PCI) drugs' effect on postoperative bleeding and the overall increase in survival rates. This outcome in turn has meant there is 


\section{Priority Paper Alerts}

New-onset atrial fibrillation is an independent predictor of in-hospital mortality in hospitalized heart failure patients: results of the EuroHeart Failure Survey.

Rivero-Ayerza M, Scholte Op Reimer W, Lenzen $\mathrm{M}$ et al.: Eur. Heart J. (2008) (Epub ahead of print).

A new study by the EuroHeart Failure Survey investigated the prognostic value of atrial fibrillation (AF) in patients admitted to hospital with heart failure. The data were designed to evaluate the effect of the various types of AF incidence on in-hospital mortality. The survey was carried out over a 6 -week period across 24 European countries in over 100 hospitals. Patients were categorized according to the type of occurrence of atrial fibrillation: AF prior to admission to hospital for heart failure, newonset $\mathrm{AF}$ diagnosed during hospitalization and no AF, either prior to or during hospitalization. Length of hospital stay and survival rates were measured and compared between groups. Patients with new-onset AF had a longer hospital stay in the intensive care unit and experienced higher mortality rates (12\%), compared with the two other groups (both $7 \%$ mortality rates). The study thus demonstrates that amongst patients hospitalized for heart failure, new-onset AF may act as an independent predictor of both duration of hospitalization and in-hospital mortality.

\section{Ceramide is a cardiotoxin in} lipotoxic cardiomyopathy.

Park TS, Hu Y, Noh HL et al.: J. Lipid Res. (2008) (Epub ahead of print).

This study implicates the accumulation of the lipotoxic molecule ceramide in lipotoxic cardiomyopathy. The heart is thought to become dysfunctional as a result of lipid overload, specifically in diabetes and obesity. The researchers investigated whether ceramide is a cardiotoxin by inhibiting de novo ceramide biosynthesis in isolated perfused LpLGPI hearts using myriocin treatment and heterozygous deletion of the $\angle C B 1$ gene, a subunit of serine palmitoyltransferase (SPT). SPT is a rate-limiting enzyme in ceramide biosynthesis and results demonstrated its inhibition had caused a reduction in fatty acid synthesis, increased glucose oxidation, more efficient systolic function and enhanced survival rates. The results of the study, therefore, suggest ceramide accumulation plays a significant role in the development of lipotoxic cardiomyopathy. The study offers a possible target for future therapies.

\section{Blood lipid test found to predict the likelihood of heart attack in women taking hormone-replacement therapy}

A new research study supported by the National Heart, Lung and Blood Institute of the NIH in the USA, has discovered that a straightforward blood test may be all that is required to determine whether an elevated risk of heart attack exists in women being treated with postmenopausal hormone therapies. Previous data on the adverse side effects of hormone therapy have demonstrated a link to a greater risk of heart attacks, clots and strokes; causing women to approach such treatment with reluctance. However, hormonereplacement therapy is still the most effective treatment for menopausal symptoms such as hot flushes and sleep disturbances, thus the decision to utilize such treatment is a difficult one for patients.

Paul F Bray, at the Jefferson Medical College of Thomas Jefferson University, PA, USA, and the lead author of the study states, "We found that a simple and widely used blood test may be useful to advise women if they are at an increased risk of a heart attack while undergoing hormone therapy ... instead of genetic testing or another more complicated test, we wanted to find a simple and effective way to assess the heart risk of hormone therapy".

The sample size utilized during the study consisted of 271 females with coronary heart disease in 40 medical facilities across the USA. In addition, a group of 707 controls were used, comprising postmenopausal women with no previous history of cardiovascular disease. Levels of blood lipids and high sensitivity $\mathrm{C}$-reactive protein (hs-CRP) were measured in the control group. The study found those entering the study with good lipid status (low-level cholesterol ratios of LDL to $\mathrm{HDL}$ ) were at no increased risk of developing heart problems whilst taking hormonereplacement therapies (either estrogen alone or estrogen plus progestin). This finding appeared to be particularly favorable to those women with a cholesterol ratio of less than 2.5. Conversely, those patients entering the study with unhealthy cholesterol levels of greater than or equal to 2.5 naturally suffered an elevated risk of developing heart-related afflictions such as coronary heart disease, which is exacerbated by taking hormone therapies.

These findings will assist physicians and their patients in making better informed choices about whether to pursue hormone therapy as a form of treatment for the menopause. Bray adds, "It should also be noted that hormones affect a number of major organs so patients must consider the totality of health risks and benefits, including coronary heart disease, stroke, venous thrombosis, breast cancer, fractures and gall bladder disease. The findings do not change the current recommendation that hormone therapy should not be used for prevention of coronary heart disease for prevention, women should focus on preventing and treating known risk factors such as high blood cholesterol and high blood pressure, and on avoiding smoking".

Source: Bray PF, Larson JC, Lacroix AZ et al.; Women's Health Initiative Investigators: Usefulness of baseline lipids and $\mathrm{C}$-reactive protein in women receiving menopausal hormone therapy as predictors of treatment-related coronary events. Am. J. Cardiol. 101(11), 1599-1605 (2008). 


\section{Study finds drug-eluting stents save both lives and money}

A new study by the University of Pennsylvania School of Medicine has discovered that the benefits of incorporating drug-eluting stents (DES) during surgery for coronary artery disease significantly outweighs those for bare-metal stents (BMS). Peter W Groeneveld, assistant professor at the University of Pennsylvania's Division of General Internal Medicine and lead author of the study, collated retrospective data on over 76,000 patients who had received a DES during 2003. The data spanning 9 months was compared with matched control data on patients who received a BMS, and found that those with DES had reduced mortality rates, a lower incidence of myocardial infarction and were less likely to require bypass surgery or follow-up procedures in the years following installation of the stent. Groeneveld stated, "There is a distinct possibility that drug-eluting stents not only reduce the need for future cardiac procedures, but also save lives".

In a related study to be published in the American Heart Journal, Groeneveld also uncovered the economic benefits of using DES over BMS. Although initially more costly, 1 year following surgery, DES were less likely to require extra surgery with only $12 \%$ requiring an additional stent compared with $15 \%$ for those with a BMS. This latter group was also twice as likely to require bypass surgery in the year following stent placement. Overall, DES were found to save an average of US\$1350 in follow-up care per patient, resulting in a saving of almost $\$ 100$ million when taking data on all patients studied into account.

Groeneveld has also postulated that further research into drug therapies could improve clinical and economic outcomes for heart patients, as treatment with drugs such as clopidogrel and cholesterol-reducing statin drugs assists considerably in reducing the complications of post-stent surgery.

Source: Groeneveld PW, Matta MA, Greenhut AP, Yang F: Drug-eluting compared with bare-metal coronary stents among elderly patients. J. Am. Coll. Cardiol. 51(21), 2017-2024 (2008).

and,

Groeneveld PW, Matta MA, Greenhut AP, Yang F: The costs of drug-eluting coronary stents among Medicare beneficiaries. Am. Heart J. 155(6), 1097-1105 (2008).

\section{New treatment guidelines for emergency response to cardiac arrest found to increase survival rates}

New treatment guidelines for responding to cardiac arrest emergencies outlined by the American Heart Association, has led to a marked improvement in survival rates. The new treatment procedures designed for use by emergency services and critical-care staff has not only resulted in a fourfold improvement in survival rates but also earlier discharge from the intensive care unit and an improvement in neurological status.

The main components of the new treatment protocol consists of sustained chest compressions with a 30:2 compression:ventilation ratio with minimal interruption, controlled ventilations, early manipulation of induced hypothermia and a quick transition of patients to specialized hospitals for postresuscitative care. Previous survival rates prior to 2005 were approximately $2.4 \%$; however following implementation of the AHA's new guidelines, this figure rose to $6.7 \%$.
The lead authors of the study, Paul Hinchey and Brent Myers of the Wake Country EMS System in North Carolina, USA, have offered the first broad analysis of the American Heart Association's guidelines, which were produced in 2005. The authors comment that, "Our findings not only demonstrate beneficial outcomes for victims of cardiac arrest, but also suggest the possibility that such treatment plans can be implemented for other medical conditions".

The older guidelines, which have been in use from January 2004 to April 2005, which recommended a 15:2 compression:ventilation ratio with no emphasis on interruption of compressions and encouraged intubation procedures, demonstrated survival rates of $2.4 \%$ overall and $12.1 \%$ for patients with ventricular fibrillation-ventricular tachycardia rhythm. However, 12 months following implementation of the new 2005 guidelines, survival rates had increased to $4 \%$ overall and to $21.8 \%$ for patients with ventricular fibrillation-ventricular tachycardia rhythm. The overall survival rates since 2005 have increased to $6.7 \%$, following full performance of the protocol.

In addition to the improvement in survival rates, the whole regime has also proved to be cost-effective, with the plan costing less than US $\$ 200$ per patient to implement. Myers commented, "All of these changes are simple, they are inexpensive, and they are incredibly effective".

Source: Hinchey P, Myers B: Out-of-hospital cardiac arrest survival after the sequential implementation of 2005 AHA guidelines for compressions, ventilations, and induced hypothermia. Abstracts of the Society of Academic and Emergency Medicine Meeting. DC, USA 29 May-1 June, 15(5), Suppl. 1, (2008) (Abstract 167). 